\title{
Constraints on Primordial Black Holes by Distortions of Cosmic Microwave Background
}

\author{
Hiroyuki Tashird* \\ Institut d'Astrophysique Spatiale, Université Paris-Sud XI, Bâtiment 121, Orsay, F-91405, France \\ Naoshi Sugiyamat \\ Department of Physics and Astrophysics, Nagoya University, Chikusa-ku, Nagoya, 464-8602, Japan and \\ Institute for Physics and Mathematics of the Universe, University of Tokyo, \\ 5-1-5 Kashiwa-no-Ha, Kashiwa City, Chiba 277-8582, Japan
}

(Dated: October 30, 2018)

\begin{abstract}
Possible influence of primordial black hole $(\mathrm{PBH})$ evaporations on cosmic microwave background $(\mathrm{CMB})$ is investigated. The spectrum distortions of CMB from the black-body spectrum are described by the chemical potential $\mu$ and the Compton parameter $y$. From COBE/FIRAS limits on $\mu$ and $y$, the power law index $n$ of primordial density fluctuations and the mass fraction of PBHs $\beta$ are constrained by employing the peak theory for the formation process of PBHs. Constraints set here are $n<1.304$ and $n<1.333$ in the thresholds of peaks $\zeta_{\text {th }}=0.7$ and $\zeta_{\text {th }}=1.2$, respectively, for the PBH mass range between $2.7 \times 10^{11} \mathrm{~g}$ and $1.6 \times 10^{12} \mathrm{~g}$, and $n<1.312$ and $n<1.343$ in the thresholds of peaks $\zeta_{\mathrm{th}}=0.7$ and $\zeta_{\mathrm{th}}=1.2$, respectively, for the PBH mass range between $1.6 \times 10^{12} \mathrm{~g}$ and $3.5 \times 10^{13} \mathrm{~g}$, which correspond to the comoving scales between $3 \times 10^{-18} \mathrm{Mpc}$ and $4 \times 10^{-17} \mathrm{Mpc}$. The constraint on the $\mathrm{PBH}$ fraction, which is the direct probe of the amplitude of density fluctuations in these scales, stays at almost the same value as $\beta<10^{-21}$ in these mass ranges. It is also found that, with these constraints, UV photons injected by PBH evaporations are unlikely to ionize the majority of hydrogen atoms.
\end{abstract}

\section{INTRODUCTION}

The properties of the primordial density fluctuations in large scales are revealed by Wilkinson Microwave Anisotropy Probe (WMAP) and galaxy redshift surveys [1, 2, 3]. The results of these observations are essentially consistent with the predictions of the inflationary model, i.e., primordial density fluctuations with an almost scale-invariant spectrum and the random Gaussian statistics.

Taking the second order terms of the inflaton potential and density perturbations into account, however, we expect to have the departure from the scale invariant spectrum and the existence of the non-Gaussian components. Therefore, precise measurements of the spectrum and the non-Gaussian components are crucial for a detailed understanding of inflation. For example, the first year WMAP data together with galaxy redshift surveys and Ly- $\alpha$ measurements preferred the non-zero running spectral index [1], while the third year data with new Ly- $\alpha$ measurements do not strongly favor the running [4].

The Ly- $\alpha$ measurements are sensitive to the power spectrum at $\sim 1 \mathrm{Mpc}$, while measurements of strong gravitational lensing have a sensitivity on much smaller scales such as $\sim 10-100 \mathrm{kpc}$. These methods are, however, limited in both scales and epochs. So far the best probe of the small scale density fluctuations is provided by the abundance of primordial black holes (PBHs) [5]. The formation of PBHs took place during the radiation dominated epoch due to the gravitational collapse of the high density region at the horizon scale when the amplitude of over-density exceeded a critical threshold. Therefore the resultant mass spectrum and the abundance of PBHs depend on the amplitude of the power spectrum for the primordial density fluctuations at the horizon crossing epoch. It is known that PBHs eventually evaporate while emitting Hawking radiation [6]. The lifetime of a $\mathrm{PBH}$ is proportional to the cubic of its mass. Therefore, PBHs with mass less than $10^{15} \mathrm{~g}$ should have evaporated away by the present epoch. When they evaporate, they emit black-body radiation and numerous kinds of particles such as neutrinos, electrons and protons.

Since PBHs with mass larger than $10^{15} \mathrm{~g}$ survive in the present universe, we can set a constraint on their abundance from the fact that PBH density cannot exceed the average matter density observed at present. We can therefore introduce $\beta(M)$ which is the fraction of the regions of mass $M$ collapsing into PBHs [7]. This can be related to the mass fraction of PBHs at the time of formation as $\beta=\rho_{\mathrm{PBH}, i} / \rho_{r, i}$ where $\rho_{\mathrm{PBH}, i}$ and $\rho_{r, i}$ are the energy densities of

*Electronic address: hiroyuki.tashiro@ias.u-psud.fr

${ }^{\dagger}$ Electronic address: naoshi@a.phys.nagoya-u.ac.jp 
$\mathrm{PBHs}$ and background radiation at the $\mathrm{PBH}$ formation epoch, respectively. The constraint on the density parameter of PBHs at present $\Omega_{\mathrm{PBH}, 0}<1$ implies $\beta<10^{-18}\left(M / 10^{15} \mathrm{~g}\right)^{1 / 2}$.

Another important constraint on the abundance of $\mathrm{PBHs}$ can be set by the phenomena of evaporation. $\mathrm{PBHs}$ emit many kinds of particles when they evaporate. Among these are diffuse gamma rays. The emitted photons from evaporated PBHs, after the recombination epoch, contribute to the diffuse gamma rays. The upper limit on diffuse gamma rays sets a stringent constraint on the abundance of $\mathrm{PBHs}$ [8]. It is also known that big bang nucleosynthesis [9] and entropy production in the early universe also provide constraints on $\mathrm{PBH}$ abundance for various mass ranges (see the review [10]).

In this paper, we investigate spectrum distortions of cosmic microwave background radiation (CMB) caused by $\mathrm{PBH}$ evaporation. Naselskii was the first to study the effect of PBHs on the recombination process and to estimate the allowed abundance of PBHs that evaporated around the recombination epoch [11]. Naselskii and Shevelev estimated distortions of $\mathrm{CMB}$ due to electrons and positrons from PBH evaporations [12]. Ricotti et al. have also investigated the effects of non-evaporating PBHs on CMB [13]. X-rays emitted by gas accretion into non-evaporating PBHs were found to modify recombination and reionization. Therefore, by studying the effect of such X-rays on the $y$-distortion and the reionization, they obtained a PBH's constraint which is larger than $10^{15} M_{\odot}$. Here, we adopt a modern analysis of CMB spectrum distortions, i.e., $\mu$ and $y$ distortions on which COBE/FIRAS has set stringent observational limits, where $\mu$ and $y$ are the chemical potential and the Compton $y$-parameter, respectively. The process we consider here is that photons which have evaporated from $\mathrm{PBHs}$ directly induce the $\mu$-distortion in $\mathrm{CMB}$ and also hit electrons in the surrounding medium. Accordingly, these electrons scatter CMB photons and generate the $y$-distortion. While electrons directly emitted by PBHs also generate the $y$-distortion via inverse Compton scattering, resultant photons are too energetic to be observed as CMB distortions in COBE/FIRAS data. Therefore we ignore electron emission from PBHs in this paper.

Distortions are created when the energy injection into CMB arises between redshift $z \sim 10^{6}$ and recombination $z \sim 1000$. Before $z \sim 10^{6}$, CMB can recover the black-body distribution via double Compton scattering or free-free emission. After recombination $(z<1000)$, there is almost no free electron to be a target of photons. Therefore we calculate $\mathrm{CMB}$ distortions caused by photons emitted by PBHs in this epoch. Consequently, we can obtain the constraint on the evaporated PBH abundance using COBE/FIRAS limits, $|\mu|<9 \times 10^{-5}$ and $y<1.5 \times 10^{-5}$ [14]. Note that the mass range of $\mathrm{PBHs}$ constrained here is between $10^{11}$ and $10^{13} \mathrm{~g}$, since this mass range corresponds to the PBHs whose lifetimes are within $1000<z<10^{6}$.

In order to estimate the number density of $\mathrm{PBHs}$, we need the threshold value of density fluctuations required for the overdense regions to collapse into PBHs. The threshold value was traditionally calculated as the critical density contrast in the simplified cosmological model by the analytic method, where the value obtained is $\delta_{c}=1 / 3$ for the radiation dominant era [5]. There has been resent progress in estimating the threshold value by employing numerical simulations based on general relativity [15, 16]. In this paper, therefore, we adopt the threshold value of Shibata and Sasaki [16].

This paper is organized as follows. In Sec. II we review the formula for evaluating the PBH mass function by using the peak theory. In Sec. III we calculate CMB distortions, $\mu$ and $y$, caused by Hawking radiation from PBHs and we set constraints on the primordial power spectral index and the PBH abundance. Conclusions are presented in Sec. IV. In this paper we assume that $h=0.70\left(H_{0}=h \times 100 \mathrm{~km} / \mathrm{s} \cdot \mathrm{Mpc}\right), \Omega_{\mathrm{b}} h^{2}=0.022$ and $\Omega_{\mathrm{M}} h^{2}=0.11$ [4]. And we set $c=\hbar=1$, where and $c$ and $\hbar$ are the speed of light and Planck's constant over $2 \pi$, respectively.

\section{PBH MASS FUNCTION}

In this section, we calculate the distribution of $\mathrm{PBHs}$ produced from the primordial density fluctuations by following the procedure of Green et al. [17]. We adopt the threshold value of density fluctuations required for the overdense regions to collapse into PBHs in the horizon crossing epoch obtained by Shibata and Sasaki [16] in which they performed numerical simulation of evolution for the metric perturbations on the uniform-expansion hyper surface. Green et al. [17] related this threshold to the gauge-invariant curvature perturbation on the uniform-density hyper surface, $\zeta$, which is defined as [18]

$$
\zeta=\mathcal{R}-H \frac{\delta \rho}{\dot{\rho}},
$$

where $\rho$ is the background density, $\delta \rho$ is the perturbed density, $\mathcal{R}$ is the curvature perturbation and dot represents the time derivative. Shibata and Sasaki's threshold depends on the environment of the PBH formation. The threshold value $\zeta_{\text {th }}$ is $\zeta_{\text {th }}=0.7$ when the density peak is surrounded by a low density region. In contrast, if the peak is surrounded by a flat Friedman-Robertson-Walker region, $\zeta_{\text {th }}=1.2$. 
Green et al. advocated the peak theory for the calculation of the PBH mass function because Shibata and Sasaki's result is described as the constraint on the peak value of fluctuations. We assume a power-law primordial power spectrum, $\mathcal{P}_{\mathcal{R}}=\mathcal{R}_{c}\left(k / k_{0}\right)^{n-1}$. From WMAP and galaxy survey results, $\mathcal{R}_{c}$ is $(24.0 \pm 1.2) \times 10^{-10}$ at the scale $k_{0}=0.002 \mathrm{Mpc}^{-1}[4]$. We smooth the density fields by a Gaussian window function with comoving size $R$. Under these assumptions, the peak theory gives the comoving number density of the peaks which are higher than $\nu$ as [17]

$$
n(\nu, R)=\frac{1}{(2 \pi)^{2}} \frac{(n-1)^{3 / 2}}{6^{3 / 2} R^{3}}\left(\nu^{2}-1\right) \exp \left(-\frac{\nu^{2}}{2}\right),
$$

where we employ the high peak limit $\nu \gg 1$. We relate $\nu$ to the threshold value of the $\mathrm{PBH}$ formation by

$$
\nu=\left[\frac{2\left(k_{0} R\right)^{n-1}}{\mathcal{R}_{c} \Gamma((n-1) / 2)}\right]^{1 / 2} \zeta_{\mathrm{th}}
$$

We can assume Eq. (2) to be the comoving number density of PBHs which are formed from the overdense regions with scale $R$.

Let us relate the smoothing scale $R$ to the PBH mass. The PBH mass depends on the initial environment around the peak as well as the threshold value. For simplicity, however, we assume that PBHs with the horizon mass are produced when the overdense regions enter the horizon. The epoch when scale $R$ crosses the horizon is evaluated as

$$
R=\frac{1}{a H}
$$

From the comoving entropy conservation, we obtain the relation between the temperature $T$ and the scale factor $a$ (which is normalized at the present epoch as $a_{0}=1$ )

$$
g_{*}^{1 / 3} a T=\text { const. }
$$

where $g_{*}$ is the number of relativistic degrees of freedom. In the radiation era, the horizon mass at horizon crossing of the comoving scale $R$ is described from Eqs. (4) and (5) as

$$
\begin{aligned}
M_{\mathrm{BH}}(R) & =\frac{4 \pi}{3}\left(\frac{8 \pi G}{3}\right)^{-1}\left[\frac{H_{0}^{2} \Omega_{\mathrm{M}}}{1+z_{\mathrm{eq}}}\left(\frac{g_{* \mathrm{eq}}}{g_{*}}\right)^{1 / 3}\right]^{1 / 2} R^{2} \\
& =10^{15}\left(\frac{g_{*}}{100}\right)^{-1 / 6}\left(\frac{R}{6.2 \times 10^{8} \mathrm{~cm}}\right)^{2} \mathrm{~g} .
\end{aligned}
$$

Here, we assume that the redshift of the matter-radiation equality is $z_{\mathrm{eq}}=3200$ [1] and the number of relativistic degrees of freedom at the equality is $g_{* \text { eq }}=3.36$.

The formation epoch of a $\mathrm{PBH}$ with the mass $M_{\mathrm{BH}}$, which we assume as the horizon crossing epoch of this mass scale, can be described in terms of the scale factor as

$$
\begin{gathered}
a_{\mathrm{BH}}\left(M_{\mathrm{BH}}\right)=\left(\frac{g_{* \mathrm{eq}}}{g_{*}}\right)^{1 / 6} H_{0} \Omega_{\mathrm{m}}^{1 / 2}\left(\frac{M_{\mathrm{BH}}}{X}\right)^{1 / 2}, \\
X=\frac{4 \pi}{3}\left(\frac{8 \pi G}{3}\right)^{-1}\left[\frac{H_{0}^{2} \Omega_{\mathrm{M}}}{1+z_{\mathrm{eq}}}\left(\frac{g_{* \mathrm{eq}}}{g_{*}}\right)^{1 / 3}\right]^{1 / 2} .
\end{gathered}
$$

Moreover, employing Eq. (6), we can rewrite Eq. (2) as a function of the PBH mass $n(\nu, M)$.

To describe the $\mathrm{PBH}$ abundance, one often uses the fraction of regions of mass $M$ which collapse to $\mathrm{PBHs}, \beta(M)$. Obviously, $\beta(M)$ also represents the mass fraction of PBHs at the time of formation. The energy density of PBHs with mass $M_{\mathrm{BH}}$ at horizon crossing of scale $R$ is expressed as $\rho_{\mathrm{BH}}\left(M_{\mathrm{BH}}\right)=M_{\mathrm{BH}} n\left(\nu, M_{\mathrm{BH}}\right) a_{\mathrm{BH}}\left(M_{\mathrm{BH}}\right)^{3}$. The mass fraction of PBHs $\beta$ can be written as

$$
\beta\left(M_{\mathrm{BH}}\right)=\frac{\rho_{\mathrm{BH}}\left(M_{\mathrm{BH}}\right)}{\rho}=\frac{1}{(2 \pi)^{2}} \frac{(n-1)^{3 / 2}}{6^{3 / 2}}\left(\nu^{2}-1\right) \exp \left(-\frac{\nu^{2}}{2}\right) .
$$

Here we adopt the high peak approximation, Eq. (2). 
One could conclude that the total number density of $\mathrm{PBHs}$ with mass $M_{\mathrm{BH}}$ at a given epoch can be described as $n\left(\nu, M_{\mathrm{BH}}\right)$. However, this is the number density of PBHs when they were formed. For the total number at a given epoch, which we express as $n_{\mathrm{BH}}\left(\nu, M_{\mathrm{BH}}, z\right)$, we need to subtract the number of PBHs which have been absorbed by larger PBHs in subsequent epochs. To take into account this effect, we consider the continuity equation for the $\mathrm{PBH}$ number density. The comoving number density of PBHs at $z$, in the range from $M_{\mathrm{BH}}$ to $M_{\mathrm{BH}}+d M_{\mathrm{BH}}$ can be described, in the high peak limit, as

$$
\begin{aligned}
n_{\mathrm{BH}}\left(\nu, M_{\mathrm{BH}}\right) d M_{\mathrm{BH}}= & -\frac{d n\left(\nu, M_{\mathrm{BH}}\right)}{d M_{\mathrm{BH}}} d M_{\mathrm{BH}} \\
= & \frac{1}{4 \pi^{2} M_{\mathrm{BH}}}\left(\frac{X(n-1)}{6 M_{\mathrm{BH}}}\right)^{3 / 2} \\
& \quad \times\left[\frac{(n-1)}{2} \nu^{4}\left(n, M_{\mathrm{BH}}\right)-\frac{3}{2}(n-3) \nu^{2}\left(n, M_{\mathrm{BH}}\right)-3\right] \exp \left(-\frac{\nu^{2}\left(n, M_{\mathrm{BH}}\right)}{2}\right) \\
\approx & \frac{1}{4 \pi^{2} M_{\mathrm{BH}}}\left(\frac{X(n-1)}{6 M_{\mathrm{BH}}}\right)^{3 / 2} \frac{(n-1)}{2} \nu^{4}\left(n, M_{\mathrm{BH}}\right) \exp \left(-\frac{\nu^{2}\left(n, M_{\mathrm{BH}}\right)}{2}\right), \\
& \quad \nu\left(n, M_{\mathrm{BH}}\right)=\left[\frac{2\left(k_{0}^{2} M_{\mathrm{BH}} / X\right)^{(n-1) / 2}}{\mathcal{R}_{c} \Gamma((n-1) / 2)}\right]^{1 / 2} \zeta_{\text {th }} .
\end{aligned}
$$

At the third step in Eq. (10), we take the high peak limit, $\nu \gg 1$.

Let us discuss the epoch of first PBH formation. For simplicity, we set the end of inflation at this epoch. Accordingly, the initial condition of $\mathrm{PBH}$ formation can be characterized by the reheating temperature. Under this assumption, the minimum mass of $\mathrm{PBHs}$ depends on this temperature. The comoving horizon scale at this epoch is expressed from Eqs. (4) and (5) as

$$
\begin{aligned}
\frac{1}{a H} & =\left[H_{0}^{2} \Omega_{\mathrm{M}}\left(1+z_{\mathrm{eq}}\right)\left(\frac{g_{*}}{g_{* \mathrm{eq}}}\right)^{1 / 3}\right]^{1 / 2}\left(\frac{T_{\mathrm{eq}}}{T_{\mathrm{rh}}}\right), \\
& =2.0 \times 10^{8}\left(\frac{g_{*}}{100}\right)^{-1 / 6}\left(\frac{T_{\mathrm{rh}}}{10^{8} \mathrm{GeV}}\right)^{-1} \mathrm{~cm},
\end{aligned}
$$

where $T_{\mathrm{rh}}$ is the reheating temperature. By using Eq. (6), we obtain the minimum mass of PBHs as

$$
M_{\mathrm{BH}, \min }\left(T_{\mathrm{rh}}\right)=9.8 \times 10^{15}\left(\frac{T_{\mathrm{rh}}}{10^{8} \mathrm{GeV}}\right)^{-2} \mathrm{~g} .
$$

In our assumption, therefore, $n(\nu, M)=0$ if $M<M_{\mathrm{BH}, \min }\left(T_{\mathrm{rh}}\right)$.

\section{CONSTRAINTS FROM THE CMB DISTORTIONS}

PBHs inject energy into CMB through Hawking radiation. In the early universe, even when there is a large amount of energy injection, CMB achieves black-body spectrum by photon-electron interaction, i.e., Compton and double Compton scatterings [19]. However, decouplings of these interactions occur one by one after $z \sim 10^{6}$ so that the distortions from the black-body are produced via the energy injection.

First, the double Compton scattering is decoupled at $z \sim 10^{6}$. After this decoupling, the total photon number between $\mathrm{CMB}$ and the injected photons conserves. Accordingly, CMB can no longer achieve the black-body spectrum if there are any photon injections after the decoupling of the double Compton scattering, although the injected photons are still thermalized by the Compton scattering. The CMB spectrum in this thermal equilibrium state is described as the Bose-Einstein spectrum with the chemical potential $\mu$ which stands for "distortions from the black-body spectrum".

Following the double Compton decoupling, the decoupling of the thermalization comes at $z \sim 10^{5}$. Due to the expansion of the universe, Compton scattering does not work effectively and can no longer establish the thermalization when the time scale of the thermalization becomes longer than the Hubble time. We must describe the distortion from the black-body spectrum due to the photon injections as the Compton- $y$ parameter once the decoupling of the thermalization takes place. 
These distortion parameters are constrained by COBE/FIRAS, $|\mu|<9 \times 10^{-5}$ and $y<1.5 \times 10^{-5}$. In this section we discussion constraints on the $\mathrm{PBH}$ abundance or the spectral index of density fluctuations by the limits of the chemical potential and the Compton- $y$ parameter.

\section{A. $\mu$-distortion}

When energy $Q$ is injected continuously into CMB, time evolution of the chemical potential, which describes the distortion of the CMB energy spectrum, is given as [20]

$$
\frac{d \mu}{d t}=-\frac{\mu}{t_{\mathrm{DC}}(z)}+1.4 \frac{Q}{\rho_{\gamma}}
$$

Here $t_{\mathrm{DC}}$ is the time scale for the double Compton scattering

$$
t_{\mathrm{DC}}=2.06 \times 10^{33}\left(1-Y_{\mathrm{p}} / 2\right)^{-1}\left(\Omega_{\mathrm{b}} h^{2}\right)^{-1} z^{-9 / 2} \mathrm{~s},
$$

where $Y_{\mathrm{p}}$ is the primordial helium mass fraction. The solution of Eq. (14) is given by Hu and Silk [20] as

$$
\begin{gathered}
\mu=1.4 \int_{0}^{t\left(z_{\text {freeze }}\right)} d t \frac{Q}{\rho_{\gamma}} \exp \left[-\left(\frac{z}{z_{\mathrm{DC}}}\right)\right], \\
z_{\mathrm{DC}}=1.97 \times 10^{6}\left(1-\frac{1}{2}\left(\frac{Y_{\mathrm{p}}}{0.24}\right)\right)^{-2 / 5}\left(\frac{\Omega_{\mathrm{b}} h^{2}}{0.0224}\right)^{-2 / 5}, \\
z_{\text {freeze }}=2.86 \times 10^{5}\left(1-\frac{1}{2}\left(\frac{Y_{\mathrm{p}}}{0.24}\right)\right)^{-1 / 2}\left(\frac{\Omega_{\mathrm{b}} h^{2}}{0.0224}\right)^{-1 / 2},
\end{gathered}
$$

where $z_{\mathrm{DC}}$ is the characteristic redshift for decoupling of the double Compton scattering and $z_{\text {freeze }}$ is the redshift when the injected energy can no longer be thermalized.

As mentioned before, the $\mu$-distortion takes place if there are any photon injections during the epoch after decoupling of the double Compton scattering and before decoupling of the Compton scattering, i.e., $z_{\text {freeze }}<z<z_{\text {DC }}$.

Let us now estimate the energy injection $Q$ due to the Hawking evaporation of PBHs. A Schwarzschild black hole with mass $M$ emits particles with spin $s$ and total energy between $E$ and $E+d E$ at a rate per degree of freedom

$$
\frac{d N_{\mathrm{emit}}}{d t d E} d E=\frac{\Gamma_{s}}{2 \pi \hbar}\left[\exp \left(\frac{E}{k T(M)}\right)-(-1)^{2 s}\right]^{-1} d E,
$$

where $T(M)$ is the temperature of a black hole with mass $M$ and written as

$$
\begin{aligned}
T(M) & =\frac{1}{8 \pi G M} \\
& \approx 1.0\left(\frac{M}{10^{13} \mathrm{~g}}\right)^{-1} \mathrm{GeV}
\end{aligned}
$$

Here $\Gamma_{s}$ is the dimensionless absorption probability of emitted species. For simplicity, we assume that only photons contribute to $Q$. In the case of photons, $\Gamma_{s}$ is described as [21]

$$
\Gamma_{s}= \begin{cases}64 G^{4} M^{4} E^{4} / 3, & E \ll k T(m), \\ 27 G^{2} M^{2} E^{2}, & E \gg k T(m) .\end{cases}
$$

The PBH mass $M$ decreases due to the Hawking evaporation. The mass loss rate of a PBH can be written as 22]

$$
\frac{d M}{d t}=-5.34 \times 10^{25} f(M) M^{-2} \mathrm{~g} \mathrm{sec}^{-1}
$$


where $f(M)$ is a function of the number of species which are directly emitted, and can be described by the fitting formula as

$$
\begin{aligned}
f(M)=1.569 & +0.569\left[\exp \left(\frac{-0.0234}{T(M)}\right)+6 \exp \left(\frac{-0.066}{T(M)}\right)+3 \exp \left(\frac{-0.11}{T(M)}\right)+\exp \left(\frac{-0.394}{T(M)}\right)\right. \\
& \left.+3 \exp \left(\frac{-0.413}{T(M)}\right)+3 \exp \left(\frac{-1.17}{T(M)}\right)+3 \exp \left(\frac{-22}{T(M)}\right)\right]+0.963 \exp \left(\frac{-0.10}{T(M)}\right) .
\end{aligned}
$$

Here $T(M)$ is written in the unit of $\mathrm{GeV}$ as in Eq. (20).

Since $f(M)$ is a weak function of $M$, we can approximately integrate Eq. (22) and obtain the time evolution of the $\mathrm{PBH}$ mass with an initial mass $M_{\mathrm{BH}}$ by

$$
M\left(M_{\mathrm{BH}}, t\right) \approx\left[M_{\mathrm{BH}}^{3}-1.5 \times 10^{26} f(M) t\right]^{1 / 3},
$$

where the typical values of $f(M)$ are $1.0,1.6,9.8$ or 13.6 for $M \gg 10^{17} \mathrm{~g}, M=10^{15} \mathrm{~g}, 10^{13} \mathrm{~g}$ or $10^{11} \mathrm{~g}$, respectively. From Eq. (22), we can also obtain the life time $\tau$ of the $\mathrm{PBH}$ with the initial mass $M_{\mathrm{BH}}$,

$$
\tau\left(M_{\mathrm{BH}}\right)=1.87 \times 10^{-27} \int^{M_{\mathrm{BH}}} d M \frac{M^{2}}{f(M)} \mathrm{s} .
$$

Finally we can write the energy injection rate due to PBHs as

$$
\dot{Q}(t)=\int_{M_{\min }(t)}^{M_{H}(t)} d M_{\mathrm{BH}} \int_{0}^{\infty} d E a^{-3}(t) n_{\mathrm{BH}}\left(M_{\mathrm{BH}}\right) \frac{d N_{\mathrm{emit}}}{d t d E}\left(M\left(M_{\mathrm{BH}}, t\right)\right) E,
$$

where $M_{H}(t)$ is the horizon mass at $t$ and $M_{\min }(t)$ is the minimum initial mass of the PBHs with a lifetime $\tau=t$. The $M_{\min }(t)$ can be evaluated by taking the inversion of Eq. (25).

Now we are ready to calculate the evolution of the chemical potential induced by photon injections from PBHs. Let us first assume that the lifetime of the smallest PBHs which are formed right after the inflation epoch is shorter than the time scale of the double Compton decoupling $t_{\mathrm{DC}}$. Under this assumption, we expect the existence of PBHs that evaporated away at the epoch of the double Compton decoupling. The mass of these PBHs is obtained as $M_{\mathrm{DC}} \equiv 2.7 \times 10^{11} \mathrm{~g}$, which corresponds to the mass scale of the horizon when the temperature $T_{\mathrm{DC}} \equiv 3.1 \times 10^{11} \mathrm{GeV}$. If the reheating temperature of inflation is higher than this temperature, i.e., $T_{\mathrm{rh}} \gg T_{\mathrm{DC}}$, the mass of the smallest PBHs formed after inflation is less than $M_{\mathrm{DC}}$.

Under this assumption, we can compute the chemical potential $\mu$ by using Eqs. (14) and (26). We show the resultant chemical potential as a function of the primordial spectral index of the power spectrum in FIG. 1) The left and right panels correspond to the cases of the critical threshold of $\mathrm{PBH}$ formation $\zeta_{\mathrm{th}}=0.7$ and $\zeta_{\mathrm{th}}=1.2$. The COBE/FIRAS upper limit on the chemical potential is $\mu<9.0 \times 10^{-5}$. We acquire the constraints on the spectral index $n$ as $n<1.304$ for $\zeta_{\text {th }}=0.7$ and as $n<1.333$ for $\zeta_{\text {th }}=1.2$.

Fig. 2 shows the production rate of the chemical potential per PBH mass. The thick solid line is the chemical potential per PBH mass for $\zeta_{\text {th }}=0.7$ and $n=1.304$ and the thick dotted line is for $\zeta_{\text {th }}=1.2$ and $n=1.333$. The vertical thin dashed line is for $M_{\mathrm{DC}}$. We can see that the majority of contributions to the chemical potential is made by PBHs with mass around $M_{\mathrm{DC}}$. This may look counterintuitive because Eq. (14) implies that, the larger the PBH mass is, the larger the chemical potential per evaporated $\mathrm{PBH}$ is produced. However the number density of PBHs with a smaller mass is much larger than that of PBHs with a larger mass. From the comparison of these two contributions, we find that PBHs with the smallest mass existing at $z_{\mathrm{DC}}$, which is $M_{\mathrm{DC}}$, provide most of the contributions for the power spectrum with $n>1$. Accordingly, PBHs with masses below $M_{\mathrm{DC}}$, which evaporated away before $z_{\mathrm{DC}}$, are not relevant for the $\mu$-distortion as is shown in Fig. 2. Since the reheating temperature only determines the minimum mass of PBHs, the constraint on the spectral index from the $\mu$-distortion does not depend on the value of the reheating temperature as far as $T_{\mathrm{rh}} \gg T_{\mathrm{DC}}$.

The other characteristic PBH mass scale is $M_{\text {freeze }} \equiv 1.6 \times 10^{12} \mathrm{~g}$, which corresponds to the mass scale of the horizon when the temperature $T_{\text {freeze }} \equiv 7.6 \times 10^{10} \mathrm{GeV}$. PBHs with $M_{\text {freeze }}$ evaporate away at the epoch of the thermalization decoupling $z_{\text {freeze }}$. This mass scale is represented as a vertical thin solid line in Fig. 2] We can see that the contribution of PBHs on the chemical potential $\mu$ is quickly suppressed once the mass exceeds $M_{\text {freeze }}$. This feature, which is represented by the kink in each thick line in Fig. 2 at $M_{\text {freeze }}$, can be easily explained by the fact that PBHs with mass below $M_{\text {freeze }}$ can only partially evaporate by $z_{\text {freeze }}$, and contribute little to the $\mu$-distortion.

Let us next consider the case of the reheating temperature $T_{\mathrm{rh}} \lesssim T_{\mathrm{DC}}$. In this case, the mass of the smallest PBHs formed after inflation, which is described as $M_{\mathrm{BH}, \min }$, is greater than $M_{\mathrm{DC}}$. Accordingly, there is almost no evaporation of PBHs at $z_{\mathrm{DC}}$. Instead, the evaporation takes place at a later epoch. Generally speaking, the lower 

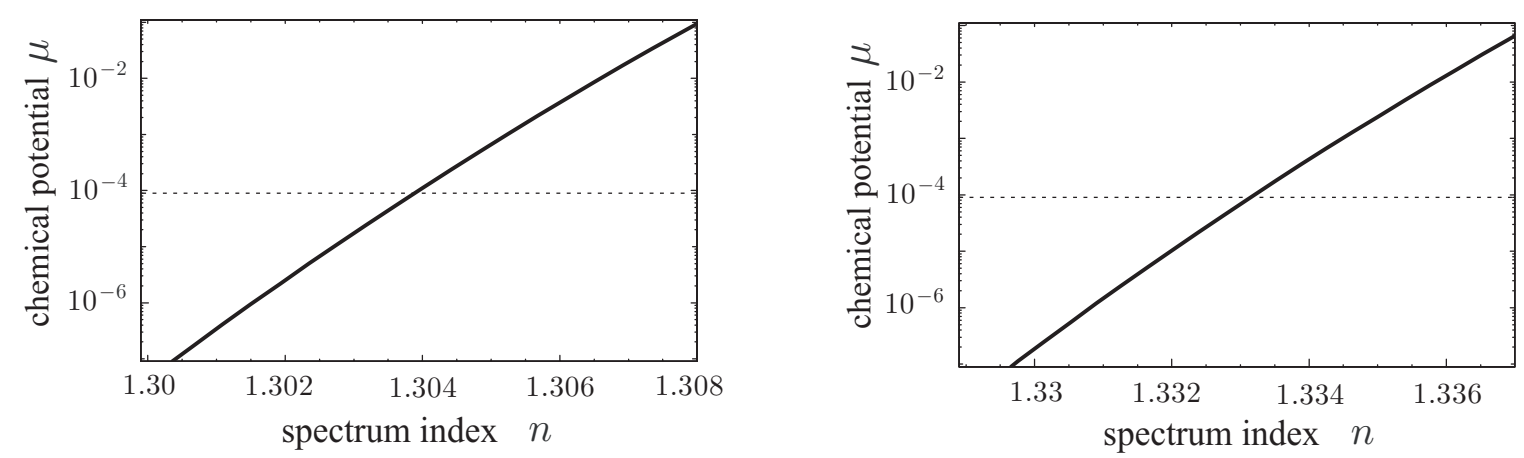

FIG. 1: The chemical potential for each spectral index. We assume the reheating temperature $T_{\mathrm{rh}} \gg T_{\mathrm{DC}}$ (see text). The critical thresholds of PBH formation are taken as $\zeta_{\text {th }}=0.7$ and 1.2 in the left and right panels, respectively. The left and right panels correspond to the cases of $\mathrm{PBH}$ formation at the density peaks surrounded by the average and low density regions, respectively. The dotted line is the upper limit of the COBE/FIRAS observation, $\mu=9.0 \times 10^{-5}$. The region under the dotted line is allowed. We find the upper limit on the spectral index $n$ as $n<1.304$ for $\zeta_{\text {th }}=0.7$ and as $n<1.333$ for $\zeta_{\text {th }}=1.2$.

the reheating temperature is, the later the PBH evaporation takes place. Therefore, we expect that the constraint on the power law index $n$ becomes looser for a lower reheating temperature. This behavior is shown in Fig. 3, Note that if the reheating temperature is so small that $M_{\mathrm{BH}, \min }\left(T_{\mathrm{rh}}\right)>M_{\text {freeze }}$, the majority of PBHs evaporate away after $z_{\text {freeze }}$. Accordingly, the constraint on the power law index becomes much looser as is shown in the left hand side of the vertical solid line of Fig. 3 .

Now, we will constrain the mass fraction of PBHs $\beta$. If $T_{\mathrm{rh}} \lesssim T_{\mathrm{DC}}$, we find that greatest contribution to $\mu$ is made by PBHs with the mass $M_{\mathrm{BH} \text {,min }}$. In this case, therefore, the constraint on the spectral index for a given reheating temperature shown in Fig. 3 can be converted into the mass fraction of the PBHs with the minimum mass. Describing the reheating temperature by $M_{\mathrm{BH}, \mathrm{min}}$ with Eq. (13), we obtain the constraint as shown in Fig. (4. On the other hand, if $T_{\mathrm{rh}} \gg T_{\mathrm{DC}}$, the most efficient PBHs for the $\mu$-distortion are those with a mass of $M_{\mathrm{DC}}$. Therefore the constraint from $\mu$-distortion does not provide sufficient clues for understanding the mass fraction on the scale below $M_{\mathrm{DC}}$. In Fig. 3, however, we extrapolate our procedure to slightly smaller masses, since contributions from masses slightly smaller than $M_{\mathrm{DC}}$ may still be important as is shown in Fig. 2. The constraint we obtain is: $\beta<10^{-21}$ between $M_{\mathrm{DC}}<M<M_{\text {freeze }}$, which is tighter than previous constraints found in [10].

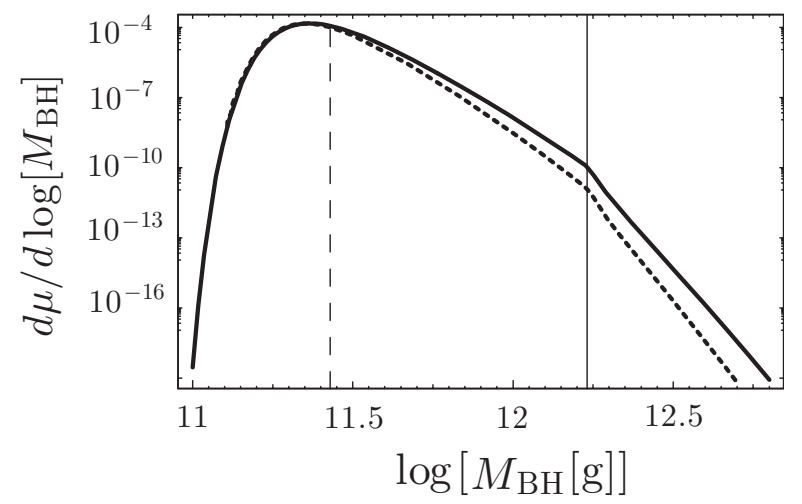

FIG. 2: The production rate of the chemical potential per PBH mass. We assume the reheating temperature $T_{\mathrm{rh}} \gg T_{\mathrm{DC}}(\mathrm{see}$ text). The thick solid and thick dotted lines correspond to the cases with $\zeta_{\text {th }}=0.7$ and $n=1.304$, and $\zeta_{\text {th }}=1.2$ and $n=1.333$, respectively. The vertical thin dashed line represents the characteristic PBH mass scale $M_{\mathrm{DC}}$ while the thin solid line represents $M_{\text {freeze }}$. It is clear that PBHs with the mass around $M_{\mathrm{DC}}$ give a dominant contribution.

\section{B. Compton $y$-parameter}

After $z_{\text {freeze }}$, the injected photons are no longer thermalized by the Compton scattering. These photons scatter electrons and the resultant energetic electrons scatter the CMB photons through the inverse Compton process. 


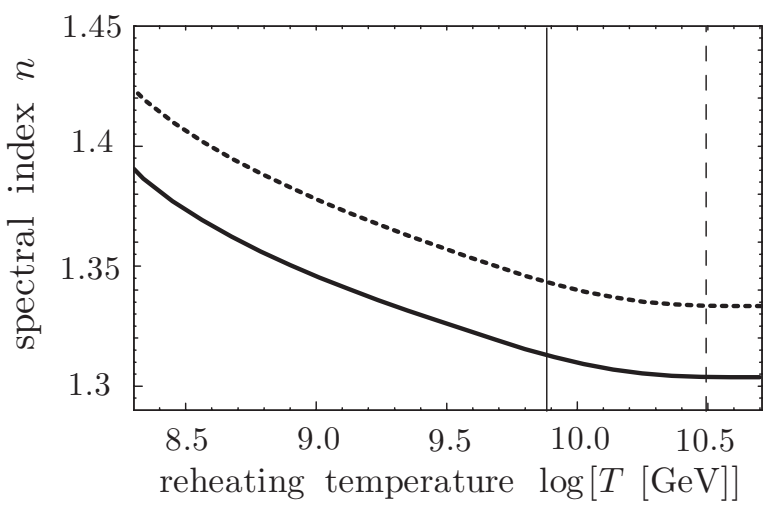

FIG. 3: The constraint on the spectral index as a function of the reheating temperature from the $\mu$-distortion. The thick solid line and the thick dotted line are the upper limits with the PBH critical threshold $\zeta_{\mathrm{th}}=0.7$ and $\zeta_{\mathrm{th}}=1.2$, respectively. The vertical thin dashed and thin solid lines represent the reheating temperatures whose horizon scales correspond to PBHs with masses $M_{\mathrm{DC}}$ and $M_{\text {freeze }}$, respectively.

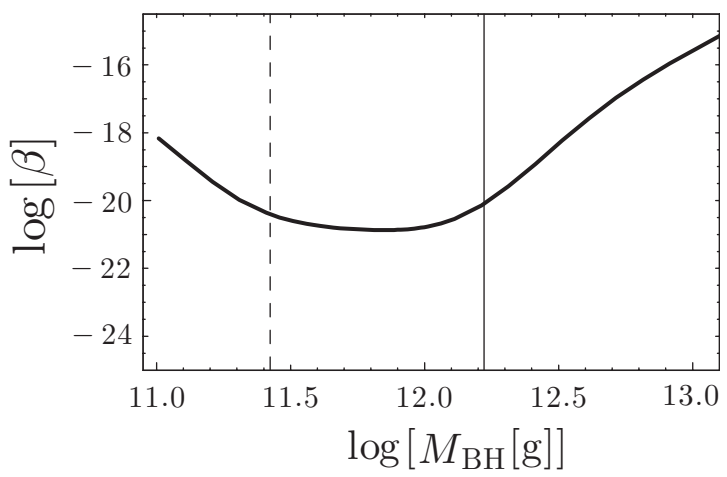

FIG. 4: The constraint on the PBH abundance from the $\mu$-distortion. The allowed region is under the thick line. The vertical thin dashed and thin solid lines represent the locations of $M_{\mathrm{DC}}$ and $M_{\text {freeze }}$, respectively.

Accordingly, Compton $y$-distortions are induced.

When $T_{e} \gg T$, where $T_{e}$ and $T$ are electron and CMB temperatures, respectively, the Compton $y$-parameter is described from the Kompaneets equation as [23]

$$
y=\int_{t\left(z_{\text {freeze }}\right)}^{t_{0}} d t \frac{k T_{e}}{m_{e}} n_{e} \sigma_{T}
$$

where $n_{e}$ and $\sigma_{T}$ are the electron number density and the Thomson scattering cross section, and $t\left(z_{\text {freeze }}\right)$ and $t_{0}$ are the freeze out time and the present time, respectively.

The electron temperature $T_{e}$ is controlled by Compton heating and Compton cooling as

$$
\begin{gathered}
3 \frac{d}{d t} k T_{e} n_{e}=n_{e} \sigma_{T} \frac{E_{\gamma}-4 k T_{e}}{m_{e}} E_{\gamma} n_{\gamma}-4 n_{e} \sigma_{T} k\left(T_{e}-T\right) \frac{\pi^{2}}{15}(k T)^{3}\left(\frac{k T}{m_{e}}\right)-15 \frac{\dot{a}}{a} k T_{e} n_{e}, \\
\frac{\partial n_{\gamma}}{\partial t}=\frac{E_{\gamma}-4 k T_{e}}{m_{e}} E_{\gamma} \frac{\partial n_{\gamma}}{\partial E_{\gamma}} n_{e} \sigma_{T}+\frac{2 E_{\gamma}-4 k T_{e}}{m_{e}} E_{\gamma} n_{\gamma} n_{e} \sigma_{T}+\frac{\dot{a}}{a} E_{\gamma} \frac{\partial n_{\gamma}}{\partial E_{\gamma}}-2 \frac{\dot{a}}{a} n_{\gamma}+\delta n_{\gamma}\left(E_{\gamma}\right),
\end{gathered}
$$

where $n_{\gamma}\left(E_{\gamma}\right)$ is the injected photon number density with the energy being from $E_{\gamma}$ to $E_{\gamma}+d E_{\gamma}$, and $\delta n_{\gamma}\left(E_{\gamma}\right)$ is the production rate of injected photons. The first term of the right hand side (rhs) of Eq. (28) describes the Compton heating of electrons by injected photons, the second term describes the Compton cooling of electrons from CMB photons and the third term describes the adiabatic cooling due to cosmic expansion. The first two terms of the rhs 
of Eq. (29) describe the Compton cooling for injected photons, and the third and forth terms describe the adiabatic cooling.

For obtaining the electron temperature $T_{e}$, we employ the analytic approach in Ref. 24]. When we assume a high electron temperature $\left(T_{e} \gg T\right)$ and the steady state $\left(d T_{e} / d t=0\right)$, we obtain the electron temperature from Eq. (28) as

$$
k T_{e}=\frac{m_{e}}{4 \rho_{\gamma}} \int_{0}^{\infty} \frac{E_{\gamma}-4 k T_{e}}{m_{e}} E_{\gamma} n_{\gamma}
$$

Here we ignore cosmic expansion.

Integrating Eq. (29) over the photon energy, we can obtain the equation for the total photon energy density,

$$
\frac{\partial}{\partial t} \int_{0}^{\infty} d E_{\gamma} E_{\gamma} n_{\gamma}=-\int_{0}^{\infty} d E_{\gamma} \frac{E_{\gamma}-4 k T_{e}}{m_{e}} E_{\gamma} n_{\gamma} n_{e} \sigma_{T}+\dot{Q},
$$

where we ignore cosmic expansion again because the time scale of the Thomson scattering is shorter than the cosmological time. The second term $\dot{Q}=\int d E_{\gamma} E_{\gamma}\left(\delta n_{\gamma}\left(E_{\gamma}\right) / d E_{\gamma}\right)$ on rhs is the total injected energy rate and is described as Eq. (26) because we consider the PBHs as the only source of the energy injection. The first term on the rhs of Eq. (31) describes the energy loss rate per unit time and, hereafter, we express this term as $E_{\text {loss }}$. From Eq. (30), we can rewrite the electron temperature in terms of $E_{\text {loss }}$ as

$$
k T_{e}=\frac{m_{e}}{4 \rho_{\gamma}} \frac{E_{\mathrm{loss}}}{n_{e} \sigma_{T}} .
$$

In order to acquire the electron temperature, we need to estimate the energy loss rate $E_{\text {loss }}$. A photon with energy $E_{\gamma}$ loses the energy $E_{\gamma}\left(E_{\gamma}-4 k T_{e}\right) / m_{e}$ per Compton scattering. Hence, the energy loss of a photon with high initial energy $E_{\gamma 0} \gg k T_{e}$ within Hubble time is approximated as

$$
\delta E_{\gamma} \simeq \frac{E_{\gamma 0}^{2}}{m_{e}} \frac{n_{e} \sigma_{T}}{H} .
$$

Substituting the Hubble time and the typical photon energy from PBHs, our present target of interested, we obtain $\delta E_{\gamma} \gg E_{\gamma 0}$. This implies that the injected photon energy is fully transferred into electrons in the Hubble time. Therefore we can approximate the energy loss rate $E_{\mathrm{loss}}$ as $E_{\mathrm{loss}} \simeq \dot{Q}$. Substituting $\dot{Q}$ for $E_{\mathrm{loss}}$ in Eq. (32), and Eq. (32) for Eq. (27), we obtain

$$
y=\int_{t\left(z_{\mathrm{freeze}}\right)}^{t\left(z_{\mathrm{rec}}\right)} d t \frac{\dot{Q}}{4 \rho_{r}}
$$

where the upper bound of the integration $t\left(z_{\text {rec }}\right)$ is the recombination epoch, which is introduced since the injected energy does not transfer into the background electrons once the optical depth becomes very low after recombination.

We calculate the $y$-distortion for each primordial power spectral index under the assumption that the reheating temperature is much higher than $T_{\text {freeze }} \equiv 7.6 \times 10^{10} \mathrm{GeV}$ which corresponds to $M_{\text {freeze }}$. The result is shown in Fig. 5. The upper limit on the $y$-parameter obtained by COBE/FIRAS is $y=1.5 \times 10^{-5}$ which is the dotted line in Fig. 5. Therefore, we obtain the constraint on the spectral index $n<1.312$ for $\zeta_{\text {th }}=0.7$ or $n<1.343$ for $\zeta_{\text {th }}=1.2$. The $y$-parameter per PBH mass is plotted in Fig. 66. The thick solid line is the $y$-parameter per PBH mass with $\zeta_{\text {th }}=0.7$ and $n=1.312$, and the thick dotted line is that with $\zeta_{\text {th }}=1.2$ and $n=1.343$. The vertical thin solid line is the location of $M_{\text {freeze }}$ and the vertical thin dotted line is $M_{\mathrm{RC}}=3.5 \times 10^{13} \mathrm{~g}$. The PBHs with $M_{\mathrm{RC}}$ evaporate away at the recombination epoch, $z \approx 1000$. Because PBHs with masses smaller than $M_{\text {freeze }}$ have evaporated away before the redshift $z_{\text {freeze }}$, they provide no contribution to the $y$-distortion. It is shown in Fig. 6 that the largest contribution to the $y$-distortion is from PBHs with mass $M_{\text {freeze }}$.

Next, we calculate the $y$-distortion in the case of the reheating temperature $T_{\text {rh }} \lesssim T_{\text {freeze }}$. We plot constraints on the power law index $n$ for a given reheating temperature in Fig. 7. The thick solid and thick dotted lines are the constraint of the spectral index for $\zeta_{\text {th }}=0.7$ and $\zeta_{\text {th }}=1.2$, respectively. The vertical thin solid and thin dotted lines represent $T_{\text {freeze }}$ and $T_{\mathrm{RC}} \equiv 1.7 \times 10^{9} \mathrm{GeV}$ which corresponds to $M_{\mathrm{RC}}$, respectively. When the reheating temperature is smaller than $T_{\text {freeze }}$, the larger spectral index is allowed because contributions on the $y$-distortion from PBHs with masses between $M_{\mathrm{BH}, \min }\left(T_{\mathrm{rh}}\right)$ and $M_{\text {freeze }}$ are missing similar to the case of the chemical potential.

We calculate the upper bound of $\beta$ from the constraint on the spectral index which is plotted in Fig. 7 We plot the result as the solid line in Fig. 8. As a reference, we plot the constraint from the chemical potential as the thick dotted line. These constraints, from $\mu$-distortion and $y$-distortion, are complementary, as is shown in the figure. On 

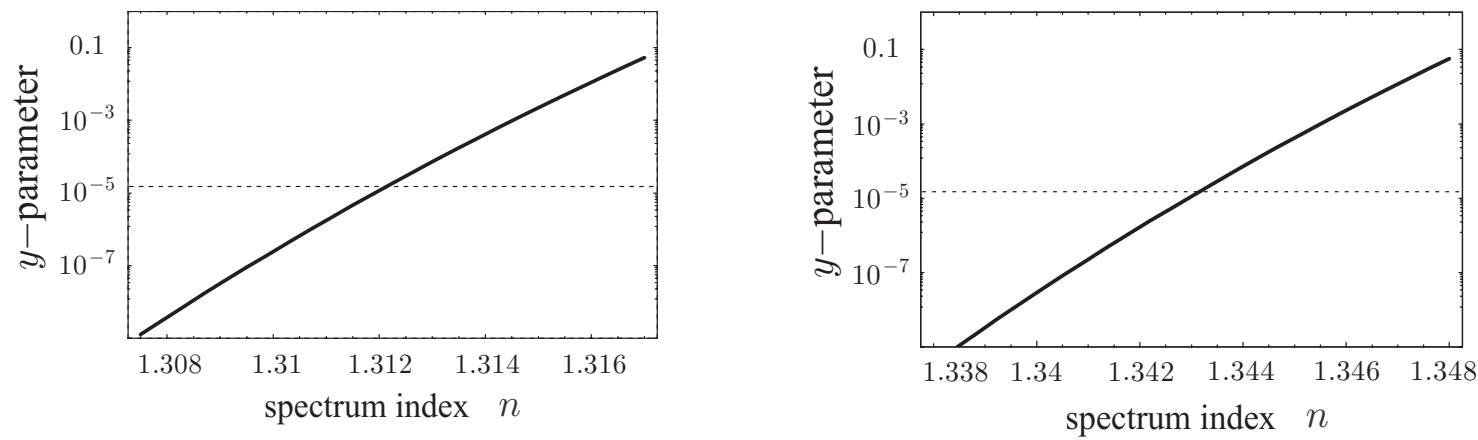

FIG. 5: The $y$-distortion as a function of the spectral index. We assume $T_{\mathrm{rh}} \gg T_{\text {freeze }}$. The critical thresholds of PBH formation are taken as $\zeta_{\text {th }}=0.7$ and 1.2 in the left and right panels, respectively. The dotted line is the upper limit of the COBE/FIRAS observation. The region under the dotted line is allowed. The upper limits on the spectral index $n$ are $n<1.312$ for $\zeta_{\text {th }}=0.7$ and $n<1.343$ for $\zeta_{\text {th }}=1.2$.

the mass scales $M_{\text {freeze }}<M_{\mathrm{BH}}$, the $y$-distortion provides a stringent constraint, while the $\mu$-distortion gives tighter constraint on $M_{\text {freeze }}>M_{\mathrm{BH}}$. We find that the upper bound of $\beta$ is $10^{-21}$ between $M_{\text {freeze }}<M<M_{\mathrm{RC}}$.

Let us explain the above constraint on $\beta$ from the $y$-distortion more intuitively. Most of the $y$-distortion is produced by PBHs with minimum mass at the redshift $z_{\text {freeze }}$. Here we denote this minimum mass as $M_{\text {mini }}$. If $T_{\text {rh }} \gg T_{\text {freeze }}$, $M_{\text {mini }}=M_{\text {freeze }}$ while $M_{\text {mini }} \gtrsim M_{\text {freeze }}$ for $T_{\text {rh }} \lesssim T_{\text {freeze. }}$. We can approximate Eq. (34) as

$$
y \approx \frac{Q\left(M_{\mathrm{mini}}\right)}{4 \rho_{r}}
$$

where $Q\left(M_{\text {mini }}\right)$ is the total injected energy between $z_{\text {freeze }}$ and $z_{\mathrm{e}}$, which describes the epoch when the PBHs with $M_{\text {mini }}$ are evaporated away. The maximum injected energy from the PBHs with $M_{\text {mini }}$ is $\rho_{\mathrm{PBH}}\left(M_{\mathrm{mini}}\right)$. The temperature at formation of PBHs with $M_{\mathrm{BH}}$ is $T_{\mathrm{BH}} \sim 3 \times 10^{9} \mathrm{GeV}\left(M_{\mathrm{BH}} / 10^{13}\right)^{1 / 2}$ from Eq. (12) so that the formation redshift leads to $z_{\mathrm{BH}} \sim 10^{22}\left(M_{\mathrm{BH}} / 10^{13}\right)^{1 / 2}$. Since $\beta$ is defined as the fraction of $\mathrm{PBHs}$ at the formation epoch, we must take into account the evolution due to cosmic expansion to estimate the PBH fraction at $z_{e}$. PBHs can be treated as a matter component while the universe is dominated by radiation. Therefore the evolution factor between $z_{\mathrm{BH}}$ and $z_{\mathrm{e}}$ can be written as $\left(z_{\mathrm{BH}}+1\right) /\left(z_{\mathrm{e}}+1\right)$. Eventually we can relate the $y$-parameter to $\beta$ as

$$
y \lesssim \frac{\rho_{\mathrm{PBH}}\left(M_{\mathrm{mini}}\right)}{4 \rho_{r}}=\frac{\Omega_{\mathrm{PBH}}}{4}=\frac{z_{\mathrm{BH}}+1}{z_{\mathrm{e}}+1} \beta,
$$

where the inequality is introduced by the fact that the maximum injected energy from PBHs provides only the upper bound for the $y$-distortion because PBHs can produce not only photons, but many kinds of particles, i.e., electrons, neutrinos, and so on. This relation is drawn in Fig. 8 as a thick dashed line. We find this intuitive approach is consistent with our previous constraint.

\section{CONSTRAINT FROM THE OPTICAL DEPTH}

In this section, we investigate the possible reionization of neutral hydrogens after recombination due to evaporation of PBHs.

We adopt the cumulative number of ionizing photons which are produced by $\mathrm{PBH}$ evaporations as the criterion of the ionization. For simplicity, we ignore the cascade decays of high energy photons which can result in a larger number of photons. If we take this effect into account, the constraint on $\beta$ which we will obtain below, shall be much tighter.

To achieve a volume-weighted ionization fraction of 99 percent, it is found that 5 to 20 cumulative photons per hydrogen atom are needed [25, 26]. The particle emission rate of PBHs is given by Eq. (19) so that the total cumulative ionizing photon number per hydrogen atom for given time $t$ is described as

$$
\frac{n_{\mathrm{cum}}}{n_{\mathrm{H}}}(t)=\frac{1}{n_{\mathrm{H}}} \int_{M_{\min }(t)}^{M_{H}(t)} d M_{\mathrm{BH}} \int_{0}^{\infty} d E n_{\mathrm{BH}}\left(M_{\mathrm{BH}}\right) \frac{d N_{\mathrm{emit}}}{d t d E}\left(M\left(M_{\mathrm{BH}}, t\right)\right),
$$

where $n_{\mathrm{H}}$ is the comoving hydrogen number density. Adopting the upper bound of the power law index $n$ from the previous section as $n \lesssim 1.3$, we find that the cumulative ionization photon number is so negligible $\left(\sim 10^{-8}\right.$ per 


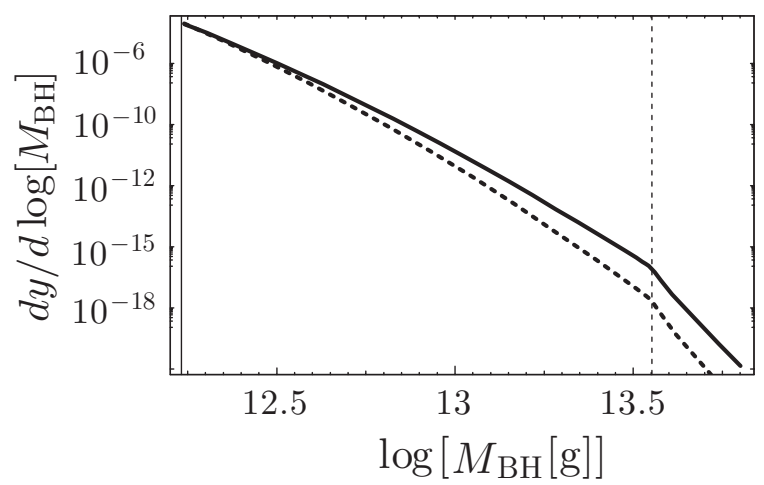

FIG. 6: The production rate of the $y$-parameter per PBH mass. We assume $T_{\mathrm{rh}} \gg T_{\text {freeze}}$. The thick solid and thick dotted lines correspond to the cases with $\zeta_{\text {th }}=0.7$ and $n=1.312$, and $\zeta_{\text {th }}=1.2$ and $n=1.343$, respectively. The vertical thin solid and dotted lines represent $M_{\text {freeze }}$ and $M_{\mathrm{RC}}$, respectively. It is shown that the contribution of the PBHs with mass $M_{\text {freeze }}$ gives a dominant contribution so that the abundance of the PBHs with the mass $M_{\text {freeze }}$.

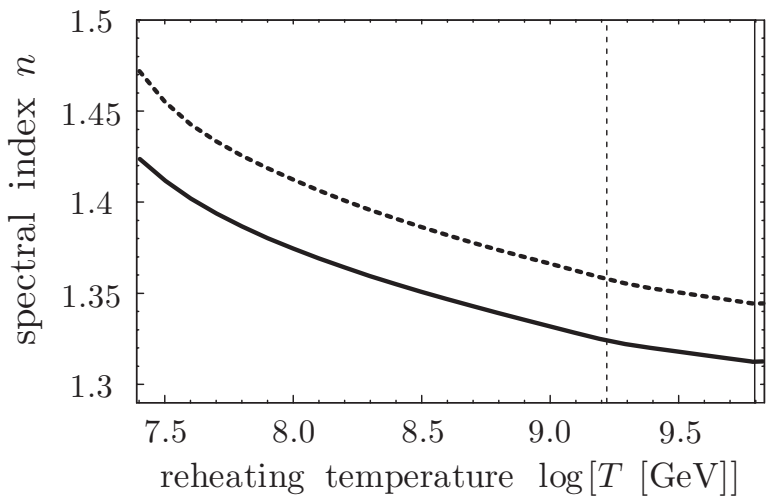

FIG. 7: The constraint on the spectral index as a function of the reheating temperature from the $y$-distortion. The thick solid line and the thick dotted line are the upper limits with the $\mathrm{PBH}$ critical threshold $\zeta_{\text {th }}=0.7$ and $\zeta_{\text {th }}=1.2$, respectively. The vertical thin dotted and thin solid lines represent the reheating temperatures whose horizon scales correspond to PBHs with masses $M_{\mathrm{RC}}$ and $M_{\text {freeze }}$, respectively.

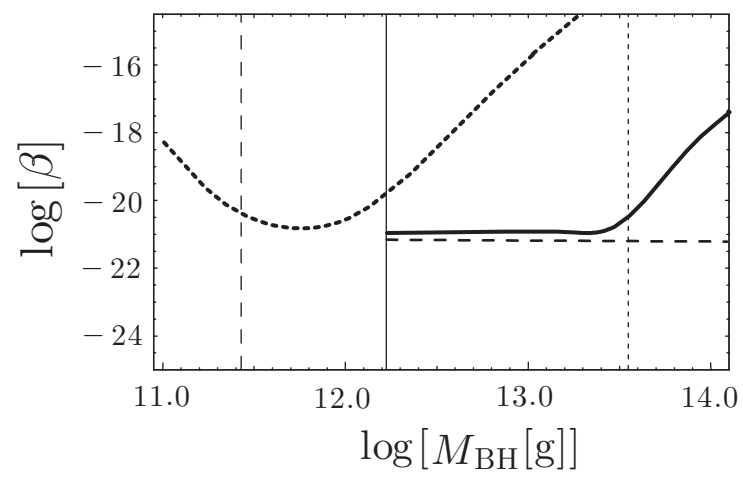

FIG. 8: The constraint on the PBH abundance from the $y$-distortion (thick solid line). The allowed region is under the thick solid line. The vertical thin solid and thin dotted lines represent the locations of $M_{\text {freeze }}$ and $M_{\mathrm{RC}}$, respectively. The thick dotted line is the constraint on the PBH abundance from the $\mu$-distortion. The thick dashed line is obtained by Eq. (36). Here we adopt the COBE/FIRAS upper limit for $y$, and describe $z_{\mathrm{e}}$ and $z_{\mathrm{BH}}$ in terms of $M_{\mathrm{BH}}$. 
hydrogen atom) that reionization cannot be caused by ionizing photons injected by PBHs unless the cascade decays of high energy photons take place very efficiently such that one energetic photon decay into, say, $10^{8}$ ionizing photons.

\section{CONCLUSION}

In this paper, we investigated the possible influence of $\mathrm{PBH}$ evaporation on $\mathrm{CMB}$. We set new and stringent constraints on the $\mathrm{PBH}$ mass fraction and the primordial power spectrum of density fluctuations from the observational upper bounds of $\mu$ and $y$-distortions obtained by COBE/FIRAS. For this analysis, we employed the peak theory, with a threshold described as $\zeta_{\text {th }}$.

It was shown that $\mu$ and $y$-distortions set limits on different ranges of PBH masses. From the $\mu$-distortion, we can test the mass range between $2.7 \times 10^{11} \mathrm{~g}$ and $1.6 \times 10^{12} \mathrm{~g}$, which correspond to $M_{\mathrm{DC}}$ and $M_{\text {freeze }}$, that is, the masses of PBHs which evaporate away at the decoupling epochs of the double Compton and the thermalization, respectively. We obtain constraints on the power law spectral index as $n<1.304$ and $n<1.333$ for $\zeta_{\text {th }}=0.7$ and $\zeta_{\text {th }}=1.2$, respectively. For the $\mathrm{PBH}$ abundance, we set the limit $\beta<10^{-21}$ for the mass range we considered here.

On the other hand, from the $y$-distortion, we investigated the $\mathrm{PBH}$ mass range between $1.6 \times 10^{12} \mathrm{~g}$ and $3.5 \times 10^{13} \mathrm{~g}$, which correspond to $M_{\text {freeze }}$ and $M_{\mathrm{RC}}$. Here $M_{\mathrm{RC}}$ corresponds to the mass of PBHs which evaporate away at the recombination epoch. We obtained constraints on the power law spectral index as $n<1.312$ and $n<1.343$ for $\zeta_{\text {th }}=0.7$ and $\zeta_{\text {th }}=1.2$, respectively. For the $\mathrm{PBH}$ abundance, we set the limit $\beta<10^{-21}$ for the mass range considered here.

It turns out that our constraints on the spectral index are looser than those obtained from the observation of CMB temperature fluctuations by WMAP satellite and other large scale observations such as galaxy surveys of $2 \mathrm{dF}$ and SDSS, and surveys of Ly- $\alpha$ forest, which imply a spectral index $n=0.947 \pm 0.015$ [1]. However, it is suggested by many authors that general inflation models produce not a simple power law spectrum but a spectrum with a running spectral index which has many branches. Accordingly it may be no use to constrain the power law index from observations on various scales. All we can do is to set constraints on the amplitude of each scale associated with the observation. From this point of view, the mass fraction of PBHs $\beta$ can provide unique information for the fluctuation amplitude on very small scales, $M_{\mathrm{DC}}<M<M_{\mathrm{RC}}$ which corresponds to the comoving scales between $3 \times 10^{-18} \mathrm{Mpc}$ and $4 \times 10^{-17} \mathrm{Mpc}$. Since these small scales correspond to the Horizon scales right after the end of inflation, we can say that the last stage of inflation can by revealed by $\mathrm{PBH}$.

Finally, we would like to mention that PBHs can be formed not only by the primordial density perturbations but also by the collisions of bubbles of the broken symmetry phase [27] or by the collapse of cosmic strings [28]. Our constraint on $\beta$ is even applicable for such PBHs.

\section{Acknowledgments}

NS is supported by a Grant-in-Aid for Scientific Research from the Japanese Ministry of Education (No. 17540276). NS thanks Institut d'astrophysique spatiale, Université Paris-Sud 11 for their kind hospitality.

[1] D. N. Spergel et al., Astrophys. J. S., 148, 175 (2003).

[2] W. J. Percival et al., Mon. Not. Roy. Astron. Soc. 327 , 1297 (2001).

[3] M. Tegmark et al., Astrophys. J. 606, 702 (2004).

[4] D. N. Spergel et al., Astrophys. J. S., 170, 377 (2007).

[5] B. J. Carr and S. W. Hawking, Mon. Not. Roy. Astron. Soc. 168 , 399 (1974).

[6] S. W. Hawking, Nature, 248, 30 (1974);

S. W. Hawking, Commun. Math. Phsy. 43, 199 (1975).

[7] B. J. Carr. Astrophys. J. 201, 1 (1975).

[8] D. Page and S. Hawking, Astrophys. J. 206, 1 (1976);

J. H. MacGibbon and B. J. Carr, Astrophys. J. 371, 447 (1991);

B. J. Carr and J. H. MacGibbon, Phys. Rep. 307, 141 (1998);

E. V. Bugaev and K. V. Konishchev, Phys. Rev. D 66, 084004 (2002).

[9] S. Miyama and K. Sato, Prog. Theor. Phys. 59, 1012 (1978);

Ya. B. Zel'dovich, A. A. Starobinsky, M. Iu. Khlopov, and V. M. Chechetkin, Pis'ma Astron. Zh. 3, 208 (1977) [Sov. Astron. Lett. 3, 110 (1977)];

D. Lindley, Mon. Not. R. Astron. Soc. 193, 593 (1980); 
T. Rothman and R. Matzner, Astrophys. Space Sci. 75, 229 (1981);

K. Kohri and J. Yokoyama, Phys. Rev. D 61, 023501 (1999).

[10] B. J. Carr and J. H. MacGibbon, Phys. Rep. 307, 141 (1998);

B. J. Carr, J. H. Gilbert and J. E. Lidsey, Phys. Rev. D 504853 (1994);

B. J. Carr and J. E. Lidsey, Phys. Rev. D 48543 (1993);

A. M. Green and A. R. Liddle, Phys.Rev. D 566166 (1997);

A. M. Green and A. R. Liddle, Phys.Rept. 307125 (1998).

[11] P. D. Naselskii, Pisma Astron. Zh. 4, 387 (1978) [Sov. Astron. Lett. 4, 209 (1978)].

[12] P. D. Naselskii and Y. G. Shevelev, Astrophysics 14, 386 (1978).

[13] M. Ricotti, J. P. Ostriker, and K. J. Mack, arXiv0709.0524 (2007).

[14] J. C. Mather et.al., Astrophys. J. 420439 (1994).

[15] J.C. Niemeyer and K. Jedamzik, Phys. Rev. Lett. 80, 5481 (1998).

[16] M. Shibata and M. Sasaki, Phys. Rev. D 60, 084002 (1999).

[17] A. M. Green, A. R. Liddle, K. A. Malik and M. Sasaki, Phys. Rev. D 70, 041502(R) (2004).

[18] D. Wands, K. A. Malik, D. H. Lyth, and A. R. Liddle, Phys. Rev. D 62, 043527 (2000).

[19] A. P. Lightman, Astrophys. J. 244, 392 (1981).

[20] W. Hu and J. Silk, Phys. Rev. D 48, 485 (1993).

[21] J. H. MacGibbon and B. R. Webber, Phys. Rev. D 41, 3052 (1990).

[22] J. H. MacGibbon, Phys. Rev. D 44, 376 (1991).

[23] Y. B. Zeldovich and R. A. Sunyaev, Ap. Space Sci., 4, 301 (1969).

[24] M. Fukugita and M. Kawasaki, Astrophys. J. 353, 384 (1990).

[25] Z. Haiman, T. Abel and P. Madau, Astrophys. J. 551, 599 (2001).

[26] A. Sokasian, T. Abel, L. Hernquist and V. Springel, Mon. Not. Roy. Astron. Soc. 344, 607, (2003);

A. Sokasian, N. Yoshida, T. Abel, L. Hernquist and V. Springel, Mon. Not. Roy. Astron. Soc. 350, 47, (2004).

[27] S. W. Hawking, I. G. Moss, J. M. Stewart, Phys. Rev. D 262681 (1982);

I. G. Moss, Phys. Rev. D 50676 (1994);

R. V. Konoplich, S. G. Rubin, A. S. Sakharov and M. Yu. Khlopov, Phys. Atom. Nucl. 621593 (1999).

[28] S. W. Hawking, Phys. Lett. B 231, 237 (1989);

A. Polnarev and R. Zembowicz, Phys. Rev. D 43, 1106 (1991);

J. Garriga and M. Sakellariadou, Phys. Rev. D 482502 (1993);

R. R. Caldwell and P. Casper, Phys. Rev. D 53, 3002 (1996);

J. H. MacGibbon, R. H. Brandenberger and U. F. Wichoski, Phys. Rev. D 572158 (1998). 